Introduction/Background* The most effective treatment strategy for FIGO stage IV endometrial cancer (EC) is currently unclear. Cytoreductive surgery is considered to improve overall survival, but this is based on small series. Further insight in the heterogeneity of this patient population's tumours and the impact of the completeness of surgery is lacking.

This study aimed to determine overall survival benefit of cytoreductive surgery in patients with FIGO stage IV endometrial cancer that underwent complete, optimal or incomplete cytoreduction compared to no surgery at all.

Methodology We performed a retrospective national multicenter cohort study analyzing retrospective data from Jan 1, 2000, to Dec 31, 2018, from patients with FIGO stage IV EC, treated in five different hospitals in The Netherlands. Patients who underwent surgery for recurrent EC or patients with a uterine sarcoma were excluded from the analysis. Kaplan Meier analysis was performed to estimate overall survival and multivariable analysis was performed with the Cox proportional hazard model. Molecular classification is currently being conducted to evaluate correlation between molecular EC class and outcome.

Result(s)* Characteristics of the 347 patients included are shown in table 1 . The most common histological subtype was endometrioid adenocarcinoma (146 of 347, 42,1\%). Complete cytoreductive surgery was achieved in 126 of 347 patients (36.3\%), while in 37 patients (10.7\%) optimal cytoreductive surgery was achieved and in 56 patients $(16,1 \%)$ incomplete cytoreductive surgery. 128 patients $(36,9 \%)$ did not receive

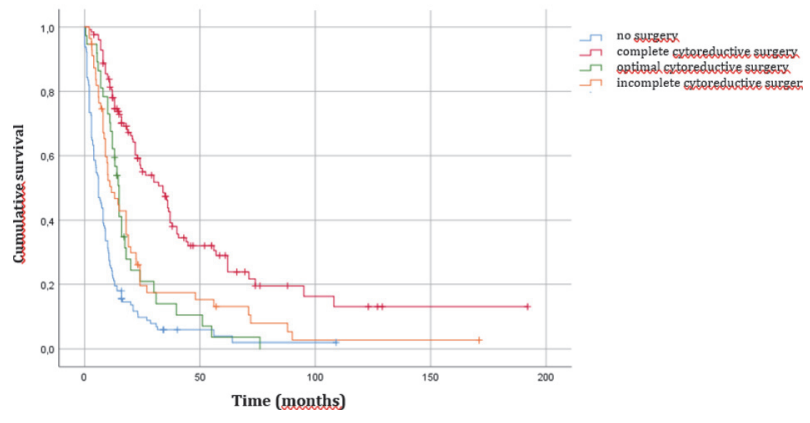

Abstract 434 Figure 1 Kaplan-Meier plots showing the overall survival curves of patients with stage IV endometrial cancer that underwent complete, optimal or incomplete cytoreductive surgery or no surgery at all

\begin{tabular}{ll} 
Abstract 434 Table 1 & Patient characteristics \\
\hline Patient characteristics (n=347) & \\
Age at diagnosis (years) & $67(33-93)$ \\
Histological subtype & $146(42.1 \%)$ \\
Endometrioid adenocarcinoma & $96(27.7 \%)$ \\
Serous carcinoma & $15(4.3 \%)$ \\
Clear cell carcinoma & $90(25.9 \%)$ \\
Other & \\
Result of surgery & $126(36.3 \%)$ \\
Complete cytoreductive surgery & $37(10.7 \%)$ \\
Optimal cytoreductive surgery & $56(16.1 \%)$ \\
Incomplete cytoreductive surgery & $128(36.9 \%)$ \\
No surgery & $13(11.1-14.9)$ \\
Overall survival in months (median) &
\end{tabular}

surgical treatment. Median overall survival was 13 months (figure 1). Complete cytoreductive surgery led to an OS of 34 months ( $p=0.00$, HR 0.34, 95\% CI: 0.26-0.45\%) compared to 15 months for patients who underwent optimal cytoreductive surgery and 11.6 months for patients who underwent incomplete cytoreductive surgery. Patients who did not receive surgical treatment had a median OS of 6 months. If possible, the molecular EC classification data will be presented at the ESGO congress.

Conclusion* Complete cytoreductive surgery leads to an extended overall survival in patients with FIGO stage IV EC in comparison to optimal or incomplete cytoreductive surgery or to no surgery at all. Our data support omission of surgical treatment when complete cytoreduction cannot be achieved.

\section{NEOADJUVANT RADIOTHERAPY FOLLOWED BY SIMPLE HYSTERECTOMY IN LOCALLY ADVANCED ENDOMETRIAL CANCER, STAGE II}

M Laseca Modrego*, O Arencibia Sanchez, D González García-Cano, AF Rave Ramirez, A Martín Martínez. Maternal Child Hospital of the Canary Islands, Oncology Gynecology Department, Las Palmas de Gran Canaria, Spain

\subsection{6/ijgc-2021-ESGO.147}

Introduction/Background* Locally advanced endometrial cancer extending to the cervix is a rare presentation of uterine cancer $(10-15 \%)$. Most of them, are stage II hidden with cervical microscopic extension discovered at the time of the pathological results. A lower percentage of stage II are diagnosed preoperatively. For the latter there, are two action plans: a) radical surgery (radical hysterectomy with bilateral salpingooophorectomy, pelvic lavage and lymphadenectomy), b) neoadjuvant radiotherapy followed by simple surgery (Simple hysterectomy with bilateral salpingo-oophorectomy).

The main objective was to know the overall survival, analyzed at 3 and 5 years, and the disease-free survival between those patients with a preoperative diagnosis of endometrial cancer stage II versus those patients with postoperative diagnosis.

Methodology Longitudinal cohort study with retrospective analysis was carried out in a third level hospital. Patients diagnosed with endometrial cancer stage II, FIGO 2009, were included. Study period 1998 to 2018.

Two cohorts were formed; women who initially received neoadjuvant radiation therapy followed by hysterectomy (preoperative diagnosis stage II) and women who received primary surgical followed by radiation (postoperative diagnosis stage II).

The overall survival, 3 and 5 years, and disease-free survival were analyzed.

Result(s)* 125 patients were included in the study. 29 patients received neoadjuvant treatment with radiotherapy and 96 patients did not receive neoadjuvant treatment. The rate of overall survival at 3 and 5 years was $78.6 \%$ in the 'neoadjuvant' cohort and $86.3 \%$ and $77.9 \%$ respectively in the 'no neoadjuvant' cohort, not finding differences statistically significant between both groups $(p=0,761)$.

No differences were in terms of disease relapses, local and distance, and in terms of disease-free survival.

Conclusion* The application of neoadjuvant radiotherapy (brachytherapy plus external radiotherapy) followed simple hysterectomy in our study population, allows to match the prognosis of patients with clinical endometrial cancer stage II, initially considered less favorable. 